\title{
ON THE $H_{p}$-PROBLEM FOR FINITE $p$-GROUPS
}

GUY T. HOGAN AND WOLFGANG P. KAPPE

1. Introduction. In $1956 \mathrm{D}$. R. Hughes [2] conjectured that if $G$ is any group, $p$ any prime, and if $H_{p}(G)$ denotes the subgroup of $G$ generated by the elements of order different from $p$, then $H_{p}(G)=1$, or $H_{p}(G)=G$, or $\left[G: H_{p}(G)\right]=p$. It has recently been shown that this conjecture is false. The counterexample constructed by G. E. Wall [6] is a finite 5-group $G$ such that $\left[G: H_{5}(G)\right]=25$. However, for the following classes of groups the $H_{p}$-conjecture is known to be true:

regular $p$-groups,

$p=2$ (Hughes [2]),

$p=3$ (Straus-Szekeres [5]),

finite groups which are not $p$-groups (Hughes-Thompson [3]),

finite $p$-groups of class at most $p$ (Zappa [7]).

In this paper we show that the $H_{p}$-conjecture is true for finite metabelian $p$-groups and finite $p$-groups with cyclic factors in the lower central series.

\section{Definitions and notation.}

$$
\begin{gathered}
{[y, x]=y^{-1} x^{-1} y x, \quad[y, 0 x]=y, \quad\left[y,{ }_{i+1} x\right]=\left[\left[y,{ }_{i} x\right], x\right],} \\
{\left[y,{ }_{i} x,{ }_{j} z\right]=\left[[y, i x],{ }_{j} z\right] .}
\end{gathered}
$$

$[A, B]=$ subgroup generated by all $[a, b]$ with $a \in A$ and $b \in B$. $G_{2}=[G, G], G_{i+1}=\left[G_{i}, G\right]$, $G^{p}=$ subgroup generated by all $g^{p}$ for $g \in G$, $\phi(G)=$ Frattini subgroup of $G$.

If $G$ is a finite $p$-group, then $\phi(G)=G_{2} G^{p}$.

$A-B=$ set of all $a \in A$ such that $a \notin B$.

$Z_{1}(G)=$ center of $G, Z_{i+1}(G) / Z_{i}(G)=Z_{1}\left(G / Z_{i}(G)\right)$.

$G$ has class $c(G) \leqq m$ if $G_{m+1}=1$. Then $G_{i} \subseteq Z_{m+1-i}(G)$.

$G$ is an ECF-group if $G / G_{2}$ has exponent $p$ and $G_{i} / G_{i+1}$ is cyclic.

$G$ is metabelian if $\left[G_{2}, G_{2}\right]=1$. For metabelian groups we write the group operation as addition.

The following identities will be used frequently:

(1) $[a, b c]=[a, c][a, b][a, b, c]$,

(2) $v^{-1} a v=a[a, v]$.

3. Lemma 1 [1, Lemma 2.9, Lemma 3.3]. Let $K$ be an ECF-group of class $c(K) \geqq p+1$. Then $K$ has subgroups $\gamma(K)$ and $\eta(K)$ with

Received by the editors May 3, 1967 and, in revised form, December 11, 1967. 
$[K: \gamma(K)]=[\gamma(K): \eta(K)]=p$ such that

$$
K_{i}=\left\langle[w, i-1 s], K_{i+1}\right\rangle
$$

and

$$
w^{p}\left[w,{ }_{p-1} s\right] \in K_{p+1}
$$

for all $s \in K-\gamma(K)$ and $w \in \gamma(K)-\eta(K)$.

Lemma 2. An ECF-group of exponent $p$ has class at most $p$.

Proof. Suppose $c(K) \geqq p+1$. Since $w^{p}=1$ Lemma 1 gives $\left[w,{ }_{p-1} s\right]$ $\in K_{p+1}$, hence $K_{p}=K_{p+1}$. But $c(K) \geqq p+1$ implies $K_{p} \neq K_{p+1}$, a contradiction.

Lemma 3. If $G$ is metabelian and $n$ an integer, then

$$
\begin{gathered}
{[u, v, w]=[u, w, v] \quad \text { for all } v, w \in G, u \in G_{2},} \\
{[v, n w]=\sum_{1 \leq i \leq n}\left(\begin{array}{c}
n \\
i
\end{array}\right)[v, i w],} \\
n(v-w)=n v+\left(\begin{array}{c}
n \\
\left.\sum_{0<i+j<n}\left(\begin{array}{c}
n \\
i+j+1
\end{array}\right)[v, i w, j v]\right)-n w .
\end{array}\right.
\end{gathered}
$$

Proof. Identities (3) and (4) follow immediately from (1) and (2). To prove (5) we observe $-n w+v-w=v+[v, n w]-(n+1) w$ and (2), so (5) follows from (3), (4) and induction on $n$.

Lemma 4 [4, III. Satz]. A finite metabelian p-group $G$ of exponent $p$ has class at most $p$. If $G$ has two generators then $c(G) \leqq p-1$.

4. Theorem. Let $G$ be a finite p-group with $H_{p}(G) \neq 1$. Then $\left[G: H_{p}(G)\right] \leqq p$ if $G$ satisfies one of the following conditions:

(a) $G_{i} / G_{i+1}$ is cyclic for all $i \geqq 2$,

(b) $G$ is metabelian.

Proof. Assume $G$ is of minimal order such that $\left[G: H_{p}(G)\right]>p$, and let $N \neq 1$ be a minimal normal subgroup of $G$. Since $G$ is a $p$ group, we have $N=\langle z\rangle, z^{p}=1$ and $z \in Z_{1}(G)$. There is some $y \in H_{p}(G)$ $\neq 1$ of order greater than $p$; hence $(y z)^{p} \neq 1, y z \in H_{p}(G)$ and $z=y^{-1}(y z)$ $\in H_{p}(G)$. Hence any minimal normal subgroup of $G$ is contained in $H_{p}(G)$. Further $G / N$ satisfies again conditions (a) or (b) because $G_{i} N / G_{i+1} N$ is a homomorphic image of $G_{i} / G_{i+1}$. Since $H_{p}(G / N)$ $\subseteq H_{p}(G) / N$, we have $\left[G / N: H_{p}(G / N)\right] \geqq\left[G: H_{p}(G)\right]>p$, and the minimality of $G$ implies $H_{p}(G / N)=1$. Hence $G / N$ has exponent $p$, so that $G^{p} \subseteq N$. Since $G^{p}=1$ implies $H_{p}(G)=1$, we have $G^{p}=N$ and $N$ is the unique minimal normal subgroup of $G$. 
(A) Assume $G$ satisfies (a). Then $G / N$ is an ECF-group since it has exponent $p$ and satisfies (a). Lemma 2 implies $c(G / N) \leqq p$, hence $c(G) \leqq p+1$, and Zappa's result [7] gives $c(G)>p$. We havc $c(G)$ $=p+1, G_{p+1}=N$ and $G$ is also an ECF-group since $N$ has order $p$. Suppose there is some $w \in \gamma(G)-\eta(G)$ such that $w \notin H_{p}(G)$. Then $w^{p}=1$ by definition of $H_{p}(G)$, and Lemma 1 gives $\left[w,{ }_{p-1} s\right] \in G_{p+1}$, hence $G_{p}=G_{p+1}$ in contradiction to $c(G)=p+1$. This proves $\gamma(G)$ $-\eta(G) \subseteq H_{p}(G)$. Now, if $x \in \eta(G)$ and $y \in \gamma(G)-\eta(G) \subseteq H_{p}(G)$, then $y x \in \gamma(G)-\eta(G) \subseteq H_{p}(G)$, hence $x=y^{-1}(y x) \in H_{p}(G)$. This proves

$$
\gamma(G)=(\gamma(G)-\eta(G)) \cup \eta(G) \subseteq H_{p}(G),
$$

and $\left[G: H_{p}(G)\right] \leqq[G: \gamma(G)]=p$ contrary to the assumption $\left[G: H_{p}(G)\right]>p$.

(B) Assume $G$ satisfies (b). Since $G / N$ has exponent $p$, Lemma 4 gives $c(G / N) \leqq p, c(G) \leqq p+1$. There is a subgroup $U$ of $G$ with $\left[U: H_{p}(G)\right]=p^{2}$. The minimality of $G$ and $H_{p}(G)=H_{p}(U)$ imply $G=U$. Hence $\left[G: H_{p}(G)\right]=p^{2}, G_{2} \subseteq H_{p}(G)$ and $G=\left\langle a, b, H_{p}(G)\right\rangle$ with $a^{p}=b^{p}=1$. Since $G^{p}=N$ and $N$ is the unique minimal normal subgroup of $G$ we see $G^{p} \subseteq G_{2}$, hence $\phi(G)=G_{2} G^{p}=G_{2}$.

(i) $[G: \phi(G)]=p^{3}$.

Let $c \in H_{p}(G)$ be an element of order greater than $p$, and $W=\langle a, b, c\rangle$. Since $H_{p}(W) \subseteq W \cap H_{p}(G)$ and $G=W H_{p}(G)$ the isomorphism theorem gives

$$
\begin{aligned}
p^{2} & =\left[G: H_{p}(G)\right]=\left[W H_{p}(G): H_{p}(G)\right] \\
& =\left[W: W \cap H_{p}(G)\right] \leqq\left[W: H_{p}(W)\right] .
\end{aligned}
$$

The minimality of $G$ and $1 \neq c \in H_{p}(G)$ imply $G=W=\langle a, b, c\rangle$, hence $[G: \phi(G)] \leqq p^{3}$. Next assume $[G: \phi(G)]=p^{2}$. Then $G_{2}=\phi(G)=H_{p}(G)$ and $c(G / N) \leqq p-1$ by Lemma 4 , hence $c(G) \leqq p$. This contradicts $\left[G: H_{p}(G)\right]>p$ either by Zappa's result [7] or directly as follows.

Since $G^{p}=N \subseteq Z_{1}(G)$, we have $0=[w, p u]=p[w, u]$ for $w \in G$ and $u \in G_{2}$. If $w \in G-H_{p}(G)$ and $v \in G_{2}=H_{p}(G)$, then $v-w \in G-H_{p}(G)$. Hence $p w=0=p(v-w)$ and (4) gives $0=p v+[v, p-1 w]$. But $c(G) \leqq p$ implies $G_{2} \subseteq Z_{p-1}(G)$, and $c\left(\left\langle w, Z_{p-1}(G)\right\rangle\right) \leqq p-1$. In particular $[v, p-1 v]=0$ and hence $p v=0$ for all $v \in G_{2}=H_{p}(G)$, a contradiction.

(ii) $G_{2}$ has exponent $p$, and all proper subgroups of $G$ have class at most $p$.

Let $W=\left\langle a, b, G_{2}\right\rangle$ and observe that $G=W H_{p}(G)$ and $H_{p}(W)$ $\subseteq W \cap H_{p}(G)$. Hence

$$
\begin{aligned}
p^{2} & =\left[G: H_{p}(G)\right]=\left[W H_{p}(G): H_{p}(G)\right] \\
& =\left[W: W \cap H_{p}(G)\right] \leqq\left[W: H_{p}(W)\right] .
\end{aligned}
$$


The minimality of $G$ and $W \neq G$ imply $H_{p}(W)=1$ and thus $W$ and $G_{2}$ have exponent $p$. Next let $V$ be a maximal subgroup of $G$. Then $V=\langle u, \quad v, \quad \phi(G)\rangle$ and $\phi(G)=G_{2} \subseteq Z_{p}(G)$ by $c(G) \leqq p+1$. But $c(\langle u, v, N\rangle / N) \leqq p-1$, by Lemma 4 , hence $c(\langle u, v\rangle) \leqq p$, and $c\left(Z_{p}(G)\langle u, v\rangle\right) \leqq p$.

(iii) $0=p z+[z, p-1 x]$ for $z \in H_{p}(G)$ and $x \in G-H_{p}(G)$.

Since $z-x \notin H_{p}(G)$ it follows from (5) and (ii) that

$$
0=p z+\sum_{i+j=p-1}[z, i x, j z] .
$$

We substitute $n x \notin H_{p}(G)$ for $x$. Since $n^{p-1} \equiv 1 \bmod p$, and $c(\langle x, z\rangle)$ $\leqq p$ by (i) and (ii), we get a system of $p-1$ equations for the quantities $\left(p z+\left[z,{ }_{p-1} x\right]\right)$ and $\left[z,{ }_{i} x,{ }_{p-1-i} z\right]$ for $i<p-1$ :

$$
0=p z+\sum_{i+j=p-1} n^{i}\left[z,{ }_{i} x, p-1-i z\right], \quad n=1, \cdots, p-1 .
$$

The determinant $\operatorname{det}\left(n^{i}\right)$ is a Vandermonde determinant and $\operatorname{det}\left(n^{i}\right)$ $\not \equiv 0 \bmod p$. Hence $0=p z+\left[z,{ }_{p-1} x\right]$ and $0=[z, i x, p-1-i z]$ for $i<p-1$.

(iv) $0=[u, i a, j b]$ for $u \in G_{2}$ and $i+j \geqq p-1$.

We substitute $u \in G_{2} \subseteq H_{p}(G)$ for $z$ and $n a+b \in G-H_{p}(G)$ for $x$ in (iii). Since $c(G) \leqq p+1$ clearly $[u, i a, j b] \in Z_{1}(G)$ for $i+j \geqq p-1$, and $p u=0$ by (ii), hence using (1) and (3) we get from (iii)

$$
0=\sum_{0<i<p} n^{i}\left(\begin{array}{c}
p-1 \\
i
\end{array}\right)\left[u,{ }_{i} a,{ }_{j} b\right], \quad i+j=p-1 ; \quad n=1, \cdots, p-1 .
$$

The Vandermonde argument gives

$$
\left(\begin{array}{c}
p-1 \\
i
\end{array}\right)[u, i a, j b]=0
$$

for $i+j=p-1$, hence (iv), since

$$
\left(\begin{array}{c}
p-1 \\
i
\end{array}\right) \not \equiv 0 \quad \bmod p
$$

(v) $0=p z$ for all $z \in H_{p}(G)$, hence $H_{p}(G)=1$, a contradiction.

We substitute $n a+b$ for $x$ in (iii). From (iv) with $u=[z, a]$ or $u=[z, b]$ we see that the terms $\left[z,{ }_{i} a,{ }_{j} b\right]$ and $\left[z,{ }_{i} b,{ }_{j} a\right]$ with $i+j \geqq p$ are trivial which occur if we expand $[z, p-1 n a+b]$ according to (1). Hence (iii) gives

$$
0=p z+\sum_{0<i<p} n^{i} X_{i}, \quad n=1, \cdots, p-1,
$$

where 


$$
X_{i}=\frac{1}{2}\left(\begin{array}{c}
p-1 \\
i
\end{array}\right)\left(\left[z,,_{i} a, p-1-i b\right]+[z, p-1-i b, i a]\right) \quad \text { for } i<p-1,
$$

and $X_{p-1}=\left[z,{ }_{p-1} a\right]+\left[z,{ }_{p-1} b\right]$. The Vandermonde argument gives in particular $0=p z+X_{p-1}$. But $a, b \notin H_{p}(G)$, so (iii) shows $0=p z$ $+[z, p-1 a]=p z+[z, p-1 b]$, hence $p z=0$.

EXAMPLE. For regular $p$-groups $H_{p}(G) \neq 1$ implies $H_{p}(G)=G$. The following example shows that conditions (a) and (b) together with $H_{p}(G) \neq 1$ do not imply $H_{p}(G)=G$. Let $A$ be an abelian group with generators $x_{1}, \cdots, x_{p-1}$ and defining relations $p^{2} x_{1}=0=p x_{2}=\cdots$ $=p x_{p-1}$. The mapping $\sigma$ defined by $x_{i}^{\sigma}=x_{i}+x_{i+1}$ for $1 \leqq i \leqq p-2$ and $x_{p-1}^{\sigma}=x_{p-1}-p x_{1}$ preserves these relations, hence $\sigma$ is an automorphism of $A$. Let $G$ be the splitting extension of $A$ by $\langle\sigma\rangle$. It follows from (4) that $\sigma$ has order $p$, and we have $G_{i}=\left\langle x_{i}, \cdots, x_{p-1}, p x_{1}\right\rangle$ for $i \leqq p-1$ and $G_{p}=\left\langle p x_{1}\right\rangle$. In particular $c(G)=p, G$ is metabelian and $G_{i} / G_{i+1}$ is cyclic for all $i$. To prove $\left[G: H_{p}(G)\right]=p$ it is sufficient to show $p(a-\sigma)=0$ for all $a \in A$ since $A \subseteq H_{p}(G)$. If $a=\alpha_{1} x_{1}+\cdots+\alpha_{p-1} x_{p-1}$, then (5) gives $p(a-\sigma)=p a+\left[a,{ }_{p-1} \sigma\right]=p \alpha_{1} x_{1}-p \alpha_{1} x_{1}=0$.

\section{REFERENCES}

1. N. Blackburn, On a special class of p-groups, Acta Math. 100 (1958), 45-92.

2. D. R. Hughes, A problem in group theory, Bull. Amer. Math. Soc. 63. (1957), 209.

3. D. R. Hughes and J. Thompson, The $H_{p}$-problem and the structure of $H_{p^{-}}$ groups, Pacific J. Math. 9 (1959), 1097-1102.

4. H. Meier-Wunderli, Metabelsche Gruppen, Comment. Helv. 25 (1951), 1-10.

5. E. G. Straus and G. Szekeres, On a problem of D. R. Hughes. Proc. Amer. Math. Soc. 9 (1958), 157-158.

6. G. E. Wall, On Hughes' $H_{p}$-problem, Proc. Internat. Conf. Theory of Groups, Austral. Nat. Univ., Canberra (August 1965), Gordon and Breach, New York, 1967.

7. G. Zappa, Contributo allo studio del problema di Hughes sui gruppi, Ann. Mat. Pura Appl. 57 (1962), 211-219.

Ohio State University 